\title{
Graded Differential Geometry in REDUCE: Supersymmetry Structures of the Modified $\mathrm{KdV}$ Equation
}

\author{
P. K. H. GRAGERT and P. H. M. KERSTEN \\ Department of Applied Mathematics, University of Twente, P.O. Box 217, 7500 AE Enschede, \\ The Netherlands
}

(Received: 30 January 1991)

Abstract. The description of a graded differential geometry package in REDUCE is given. The procedures are useful in the study of supersymmetric equations. The supersymmetric modified $\mathrm{KdV}$ equation is discussed as an application.

AMS subject classifications (1991). 35Q53, 35A30, 58G05, 58G35.

Key words. Computer algebra, graded differential geometry supersymmetries, conserved quantities.

\section{Introduction}

The interest of mathematical physicists in prolongation structures and symmetries has led to the construction of computer algebra programs to handle the massive computations arising from these problems on the computer.

Many problems have been handled using these programs [1,2] and the reader is referred to them for further references. Schwarz [3] constructed a program which deals with the determination of point symmetries of differential equations, a program in the symbolic language REDUCE [4] which runs automatically.

Recently, Reid [5] constructed a program in Macsyma to construct the structure of the point symmetry algebra of equations without integration. A program constructed by Chanpagne et al. [6] is similar to [3] and has the disadvantage of being very time consuming due to expressionswell.

The introduction of the concept of supersymmetry [7], treating bosonic and fermionic fields on an equal footing, boiled down to the mathematical concept of graded differential geometry [8] and stimulated the authors to the subject of the present research.

In Section 1, we shall set down the general notions of graded differential geometry which have been taken from [8].

In Section 2, we give a general description of the procedures which have been constructed. For a more detailed description of the procedures, the reader is referred to [9].

In Section 3 we give an application of the developed software to construct bosonic 
and fermionic symmetries and conservation laws for the Manin-Radul supersymmetric modified Korteweg-de Vries equation. For other results obtained by the computer programs described below we refer to [13] where we derived higher-order symmetries, recursion operators, and higher-order supersymmetries and fermionic conservation laws for the $\mathrm{KdV}$ equation.

Roelofs and Van den Hijligenberg constructed a graded Lie algebra prolongation of the supersymmetric KdV equation [14]. Roelofs and Kersten [15] constructed supersymmetric extensions of the nonlinear Schrodinger equation and discussed the symmetry structure and prolongation algebra.

\section{Basic Notions of Graded Differential Geometry}

In this section, we give a short review of the notions of graded differential geometry which are needed for implementation on the computer by means of the procedure described in the next sections, i.e. graded commutative algebra, graded Lie algebra, graded manifold, graded derivation, graded vector field, graded differential form, exterior differentiation, interior differentiation (or contraction) by a vector field, and Lie differentiation by a vector field.

The notions and notations have been taken from Kostant [8] and the reader is referred to this paper for more details.

Throughout this section, the field is $\mathbb{B}$ or $\mathbb{C}$ and the grading will be with respect to $\mathbb{Z}_{2}=\{0,1\}$.

1. A vector space $V$ over $\mathbb{R}$ is a graded vector space if one has $V_{0}, V_{1}$ subspaces of $V$, such that

$$
V=V_{0}+V_{1}
$$

is a direct sum.

Elements of $V_{0}$ are called even, elements of $V_{1}$ are called odd; elements of $V_{0}$ or $V_{1}$ are called homogeneous elements. If $v \in V_{i}(i=1,1)$, then $i$ is called the degree of $v$, i.e.

$$
|v|=i \quad\left(i=0,1 ; i \in \mathbb{Z}_{2}\right)
$$

(Convention: | | is used for homogeneous elements only.)

2. $A$ graded algebra $B$ is a graded vector space $\left(B=B_{0}+B_{1}\right)$ such that

$$
B_{i} B_{j} \subset B_{i+j} \quad\left(i, j=0,1 \in \mathbb{Z}_{2}\right) \text {. }
$$

3. A graded algebra $B$ is called graded commutative if for any two homogeneous elements $x, y \in B$ we have

$$
x y=(-1)^{|x|}|y| y x
$$

4. $V$ is a left module for the graded algebra $B$ if $V$ is a left module in the usual sense but $V$ is also a graded vector space $\left(V=V_{0}+V_{1}\right)$ and

$$
B_{i} \cdot V_{j} \subseteq V_{i+j} \quad\left(i, j \in \mathbb{Z}_{2}\right)
$$


the right modules are defined similarly.

5. If $V$ is a left module for the graded commutative algebra $B$, then $V$ inherits a right module structure where we define

$$
v \cdot b=(-1)^{|b||v|} b \cdot v \quad(v \in V ; b \in B) .
$$

Similarly, a left module structure is defined by a right module structure.

6. A graded vector space $\mathbf{g}=\mathbf{g}_{0}+\mathbf{g}_{1}$, together with a bilinear operation $[*, *]$ on $\mathbf{g}$ such that $[x, y] \in \mathbf{g}_{|x|+|y|}$ is called a graded Lie algebra (GLA) if

(1) $[x, y]=-(-1)^{|x||y|}[y, x]$,

(2) $(-1)^{|x||z|}[x,[y, z]]+(-1)^{|z||y|}[z,[x, y]]+(-1)^{|y||x|}[y,[z, x]]=0$.

$(1.7 \mathrm{~b})$ is called the (graded) Jacobi identity.

If $V$ is a graded vector space, then $\operatorname{End}(V)$ has the structure of a GLA by

$\alpha, \beta \in \operatorname{End}(V) \Rightarrow[\alpha, \beta]=\alpha \beta-(-1)^{|\alpha| \beta \beta \mid} \beta \alpha$.

7. If $B$ is a graded algebra, an operator $\alpha \in \operatorname{End}(B)_{i}$ is called a (graded) derivation of $B$ if

$$
\alpha(x y)=\alpha(x) \cdot y+(-1)^{|i||x|} x \cdot \alpha(y) .
$$

An operator $\alpha \in \operatorname{End}(B)$ is a derivation if its homogeneous components are so. The graded vector space of derivations of $B$, denoted $\operatorname{Der}(B)$ is a graded Lie subalgebra of $\operatorname{End}(B)$. (1.9) is called the (graded) Leibniz rule.

If $B$ is a graded commutative algebra, then $\operatorname{Der}(B)$ is a left $B$-module where if $\zeta \in \operatorname{Der}(B), f, g \in B$ then $f \zeta \in \operatorname{Der}(B)$, where

$$
(f \zeta) g=f(\zeta g) \text {. }
$$

8. The local picture of a graded manifold is $U \subset \mathbb{R}^{m}$ open together with the graded commutative algebra

$$
C^{\infty}(U) \otimes \Lambda(n)
$$

where $\Lambda(n)$ is the antisymmetric (exterior) algebra on $n$ elements

$$
s_{1}, \ldots, s_{n},\left|s_{i}\right|=1 \quad(i=1, \ldots, n) ; \quad s_{i} s_{j}=-s_{j} s_{i}(i, j=1, \ldots, n) .
$$

A particular element $f \in C^{\infty} \otimes \Lambda(n)$ is represented as

$$
f=\sum_{\mu} f_{\mu} s_{\mu}
$$

where

$$
\begin{aligned}
& \mu \in M_{n}=\left\{\mu=\left(\mu_{1}, \ldots, \mu_{k}\right) \mid \mu_{i} \in \mathbb{N}, 1 \leqslant \mu_{1}<\mu_{2} \cdots<\mu_{k} \leqslant n\right\}, \\
& s_{\mu}=s_{\mu_{1}} \cdots s_{\mu_{2}}, \quad f_{\mu} \in C^{\infty}(U),
\end{aligned}
$$

the elements $s_{\mu}$ will be represented by $\operatorname{ALT}\left(\mu_{1}, \mu_{2}, \ldots, \mu_{k}\right)$ in the system (cf. Section 2). 
9. Graded vector fields on a graded manifold $\left(U, C^{\infty}(U) \otimes \Lambda(n)\right)$ are introduced as graded derivations of the algebra $C^{\infty}(U) \otimes \Lambda(n)$; they constitute a left $C^{\infty}(U) \otimes \Lambda(n)$-module. Locally, a graded vector field $V$ is represented as

$$
V=\sum_{i=1}^{m} f_{i} \frac{\partial}{\partial r_{i}}+\sum_{j=1}^{n} g_{j} \frac{\partial}{\partial s_{j}},
$$

where $f_{i}, g_{j} \in C^{\infty}(U) \otimes \Lambda(n), r_{i}(i=1, \ldots, m)$ is a local coordinate system on $U \subset \mathbb{R}^{m}$.

The derivations $\partial / \partial r_{i}(i=1, \ldots, m)$ are even, while the derivations $\partial / \partial s_{j}$ are odd, they satisfy the relations

$$
\begin{aligned}
& \frac{\partial}{\partial r_{i}} r_{k}=\delta_{i k} ; \quad \frac{\partial}{\partial r_{i}} s_{j}=0 ; \quad \frac{\partial}{\partial s_{j}} r_{i}=0 ; \quad \frac{\partial}{\partial s_{j}} s_{l}=\delta_{j l} \\
& (i, k=1, \ldots, m, j, l=1, \ldots, n)
\end{aligned}
$$

10. A graded differential ( $k$-) form is introduced as a $k$-linear map $\beta$ on $\operatorname{Der}\left(C^{\infty}(U) \otimes \Lambda(n)\right)$ which has to satisfy

$$
\left\langle\zeta_{1}, \ldots, f \zeta_{l}, \ldots, \zeta_{k} \mid \beta\right\rangle=(-1)^{|f| \Sigma_{k=1}^{i-1}\left|\zeta_{i}\right|} f\left\langle\zeta_{1}, \ldots, \zeta_{k} \mid \beta\right\rangle
$$

and

$$
\left\langle\zeta_{1}, \ldots, \zeta_{j}, \zeta_{j+1}, \ldots, \zeta_{k} \mid \beta\right\rangle=(-1)^{1+\left|\zeta_{j}\right| \zeta_{j+1} \mid\left\langle\zeta_{1}, \ldots, \zeta_{j+1}, \zeta_{j}, \ldots, \zeta_{k} \mid \beta\right\rangle}
$$

for all

$$
\zeta_{i} \in \operatorname{Der}\left(C^{\infty}(U) \otimes \Lambda(n)\right) \quad \text { and } \quad f \in C^{\infty}(U) \otimes \Lambda(n) .
$$

The set of $k$-forms is denoted by $\Omega^{k}(U)$.

Note: Actually we had to write $\Omega^{k}\left(U, C^{\infty}(U) \otimes \Lambda(n)\right)$, but we have made our choice for the abbreviated notation $\Omega^{k}(U)$.

$\Omega^{k}(U)$ has the structure of a (right) $C^{\infty}(U) \otimes \Lambda(n)$-module by

$$
\left\langle\zeta_{1}, \ldots, \zeta_{k} \mid \beta f\right\rangle=\left\langle\zeta_{1}, \ldots, \zeta_{k} \mid \beta\right\rangle f \text {. }
$$

One puts

$$
\Omega^{0}(U)=U^{\infty}(U) \otimes \Lambda(n) \quad \text { and } \quad \Omega(U)=\bigoplus_{k=0}^{\infty} \Omega^{k}(U) .
$$

Moreover, $\Omega(U)$ can be given the structure of a bigraded $\left(\mathbb{Z}_{+}, \mathbb{Z}_{2}\right)$ commutative algebra, that is if $\beta_{i} \in \Omega^{k_{i}}(U)_{j_{i}}(i=1,2)$ then

$$
\beta_{1} \beta_{2} \in \Omega^{k_{1}+k_{2}}(U)_{j_{1}+j_{2}}
$$

and

$$
\beta_{1} \beta_{2}=(-1)^{k_{1} k_{2}+j_{1} j_{2}} \beta_{2} \beta_{1} .
$$

(For a general definition of $\beta_{1} \beta_{2}$, see [8].) 
One defines the exterior derivative

$$
\begin{aligned}
d: \Omega^{0}(U) & \rightarrow \Omega^{1}(U) \\
f & \rightarrow \mathrm{d} f
\end{aligned}
$$

by the condition

$$
\langle\zeta \mid \mathrm{d} f\rangle=\zeta f
$$

for $\zeta \in \operatorname{Der}\left(C^{\infty}(U) \otimes \Lambda(n)\right)$ and $f \in \Omega^{0}(U)=C^{\infty}(U) \otimes \Lambda(n)$.

By (1.18), (1.19) and the definitions of $\beta_{1} \beta_{2}$ (see [8], pp. 245, 246)

$$
\mathrm{d} r_{i} \quad(i=1, \ldots, m), \quad \mathrm{d} s_{j} \quad(j=1, \ldots, n)
$$

defined by

$$
\begin{array}{ll}
\left\langle\frac{\partial}{\partial r_{k}} \mid \mathrm{d} r_{i}\right\rangle=\delta_{i k}, & \left\langle\frac{\partial}{\partial s_{j}} \mid \mathrm{d} r_{i}\right\rangle=0 \\
\left\langle\frac{\partial}{\partial r_{k}} \mid \mathrm{d} s_{l}\right\rangle=0, & \left\langle\frac{\partial}{\partial s_{j}} \mid \mathrm{d} s_{l}\right\rangle=\delta_{j l}
\end{array}
$$

generates $\Omega(U)$ and $\beta \in \Omega(U)$ can be uniquely written as

$$
\beta=\sum_{\mu, \nu} \mathrm{d} r_{\mu t} \mathrm{~d} s^{\nu} f_{\mu, v},
$$

where

$$
\begin{aligned}
& \mu=\left(\mu_{1}, \ldots, \mu_{k}\right) \quad 1 \leqslant \mu_{1}<\cdots<\mu_{k} \leqslant n, l(\mu)=k, \\
& v=\left(v_{1}, \ldots, v_{n}\right) \quad v_{i} \in N=\mathbb{Z}_{+} \backslash\{0\}, \quad|v|=\sum_{i=1}^{n} v_{i}, \\
& f_{n, v} \in C^{\infty}(U) \otimes \Lambda(n) .
\end{aligned}
$$

Note, in particular, that by $(1.18)$

$$
\mathrm{d} s_{j} \mathrm{~d} s_{k}=\mathrm{d} s_{k} \mathrm{~d} s_{j}
$$

and by consequence

$$
\left(\mathrm{d} s_{j}\right)^{v_{j}}=\mathrm{d} s_{j} \cdots \mathrm{d} s_{j} \neq 0, \quad v_{j} \text { times. }
$$

By means of $(1.21) \mathrm{d}: \Omega^{0}(U) \rightarrow \Omega^{1}(U)$ has the following explicit representation

$$
\mathrm{d} f=\sum_{i=1}^{m} \mathrm{~d} r_{i} \frac{\partial f}{\partial r_{i}}+\sum_{j=1}^{n} \mathrm{~d} s_{j} \frac{\partial f}{\partial s_{j}} .
$$

11. Since $\Omega(U)$ is a bigraded commutative algebra $\left(\mathbb{Z}_{+}, \mathbb{Z}_{2}\right), \operatorname{End}(\Omega(U))$ is bigraded too and if $u \in$ End $\Omega(U)$ is of bidegree $(b, j) \in\left(\mathbb{Z}_{+}, \mathbb{Z}_{2}\right)$ then

$$
u\left(\Omega^{a}(U)_{i}\right) \in \Omega^{a+b}(U)_{i+j} .
$$

Now $u \in \operatorname{End} \Omega(U)$ of bidegree $(b, j)$ is a (bigraded) derivation of $\Omega(U)$ if for any 


$$
\begin{aligned}
& \alpha \in \Omega^{a}(U)_{i} k, \beta \in \Omega(U) \\
& u(\alpha \beta)=u(\alpha) \beta+(-1)^{a b+i j} \alpha u(\beta)
\end{aligned}
$$

(Leibniz rule).

There exists a unique derivation (exterior differentiation)

$$
\mathrm{d}: \Omega(U) \rightarrow \Omega(U)
$$

of bidegree $(1,0)$, such that

(1) $\left.\mathrm{d}\right|_{\Omega^{\circ}(U)}$ is defined by $(1.19),(1.24)$,

(2) $\mathrm{d}^{2}=0$.

If $\beta \in \Omega(U)$

$$
\beta=\sum_{\mu, v} \mathrm{~d} r_{\mu} \mathrm{d} s^{v} f_{\mu, v}
$$

then

$$
\mathrm{d} \beta=\sum_{u, v}(-1)^{(\mu)+|n|} \mathrm{d} r_{\mu} \mathrm{d} s^{\nu} \mathrm{d} f_{\mu, v} .
$$

Other familiar operations on ordinary manifolds have their counter parts in the graded case.

$\zeta \in \operatorname{Der}\left(C^{\infty}(U) \otimes \Lambda(n)\right)$ is an interior differentiation by $\zeta$ (or contraction by $\left.\zeta\right), i(\zeta)$ defined by

$$
\left\langle\zeta_{1}, \ldots, \zeta_{b} \mid i(\zeta) \beta\right\rangle=(-1)^{\left.|\zeta|\right|_{i=1} ^{b}\left|\zeta_{i}\right|}\left\langle\zeta, \zeta_{1}, \ldots, \zeta_{b} \mid \beta\right\rangle
$$

for $\zeta, \zeta_{1}, \ldots, \zeta_{b} \in \operatorname{Der}\left(C^{\infty}(U) \otimes \Lambda(n)\right)$, and $\beta \in \Omega^{b+1}(U)$.

Moreover

$$
i(\zeta): \Omega(U) \rightarrow \Omega(U) \quad\left(\beta \in \Omega^{b+1}(U), \quad i(\zeta) \beta \in \Omega^{b}(U)\right)
$$

is a derivation of bidegree $(-1,|\zeta|)$.

The bigraded derivations on $\Omega(U)$ can be shown to constitute a bigraded Lie algebra $\operatorname{Der} \Omega(U)$ by the following Lie bracket if $u_{1}, u_{2} \in \operatorname{Der} \Omega(U)$ of bidegree $\left(b_{i}, b_{j}\right)(i=1,2)$ then

$$
\left[u_{1}, u_{2}\right]=u_{1} u_{2}-(-1)^{b_{1} b_{2}+j_{1} j_{2}} u_{2} u_{1} \in \operatorname{Der} \Omega(U)
$$

(Lemma 4.3.2 of [8]).

From (1.29), we have that Lie differentiation by the vector field $\zeta$,

$$
L_{\zeta}=\mathrm{d} i(\zeta)+i(\zeta) \mathrm{d}
$$

is a derivation of $\Omega(U)$ of bidegree $(0,|\zeta|)$.

The fact that exterior differentiation (d), interior differentiation by $\zeta(i(\zeta))$, and Lie differentiation by $\zeta\left(L_{\zeta}\right)$ are derivations, will be used to implement them on the computer starting from the representation of vector fields and differential forms (1.14), (1.22). 


\section{General Description of the Procedures}

A general description of the procedures, constituting the graded differential geometry package is given. The source code of the procedures is given in [9]. We list below some global data required by the package

D!@DIF,DIMODDV-DIMEVENV

OPERATOR VNAT, EVENLVN, ODDLVN, SUPMULNAME , !@VECVAR OPERATOR ALT, WEDGE.

Note. Since the construction is effectively based on an extension of the ordinary differential geometry package described in [1], we shall often refer to the procedures described in that paper.

We shall first discuss the representations of graded functions, graded vector fields, and graded differential forms as algebraic objects.

\subsection{REPRESENTATION OF GRADED FUNCTIONS}

Since a graded function $f$ is an element of the graded commutative algebra $C^{\infty}\left(\mathbb{R}^{m}\right) \otimes \Lambda(n)$

$$
f \in C^{\infty}\left(\mathbb{R}^{m}\right) \otimes \Lambda(n)
$$

and ordinary differential forms can be considered as such with a slight modification, we take the following representation (see [1]).

$$
f=\sum_{\mu} f_{\mu} s_{\mu} \quad(1.13) \leftrightarrow f=\sum F_{\mu}{ }^{*} \operatorname{ALT}\left(\mu_{1}, \ldots, \mu_{k}\right)
$$

where $\mu$ is a multi-index

$$
\mu \in M_{n}=\left\{\mu=\left(\mu_{1}, \ldots, \mu_{k}\right) \mid \mu_{i} \in \mathbb{N}, \quad 1 \leqslant \mu_{1}<\cdots<\mu_{k} \leqslant n\right\} .
$$

Note that the global variables DIMEVENV, DIMODDV are just

DIMEVENV $:=m$

DIMODDV $:=n$.

EXAMPLE 2.1. $f=f_{1} s_{1}+f_{2} s_{1} s_{2}$ is represented as

$$
f:=\mathrm{F}(1)^{*} \mathrm{ALT}(1)+\mathrm{F}(2)^{*} \mathrm{ALT}(1,2) \$
$$

whereas the dependency of $F\left({ }^{*}\right)$ is given on the DEPendency List DEPL?*

$$
\text { DEPL!*:=(((f 1) R1 R2 ... RM) ((F 2) R1 R2 .. RM))\$ }
$$

\subsection{REPRESENTATION OF GRADED VECTOR FIELDS}

A graded vector field or a derivation of the graded commutative algebra 
$C^{\infty}\left(\mathbb{R}^{m}\right) \otimes \Lambda(n)$ has a local coordinate presentation

$$
V=\sum_{i=1}^{m} a_{i} \frac{\partial}{\partial r_{i}}+\sum_{j=1}^{n} b_{j} \frac{\partial}{\partial s_{j}},
$$

where $a_{i}, b_{j} \in C^{\infty}\left(\mathbb{R}^{m}\right) \otimes \Lambda(n)(i=1, \ldots, m ; j=1, \ldots, n)$.

Note. In any case, the left module structure for the graded vector fields will be assumed and used.

For ordinary vector fields [2] we took the polynomial representation

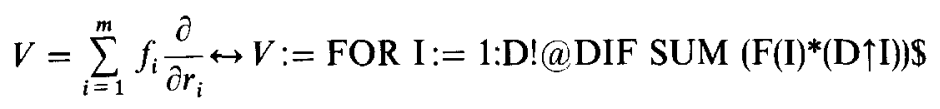

where the global variable D!@DIF has the value of the dimension of the manifold; or

$$
\frac{\partial}{\partial r_{i}} \leftrightarrow D \uparrow I \quad(i, I=1, \ldots, m) .
$$

For graded vector fields, we take a similar representation taking care of the even and odd variables, i.e.

$$
\begin{aligned}
V= & \sum_{i=1}^{m} a_{i} \frac{\partial}{\partial r_{i}}+\sum_{j=1}^{n} b_{j} \frac{\partial}{\partial s_{j}} \leftrightarrow \\
V:= & \left(\text { FOR I }:=1: \text { DIMEVENV SUM }\left(\mathrm{A}(\mathrm{I})^{*} \mathrm{DR} \uparrow \mathrm{I}\right)\right)+ \\
& \left(\text { FOR J }:=1: \text { DIMODDV SUM }\left(\mathrm{B}(\mathrm{J})^{*} \mathrm{DS} \uparrow \mathrm{J}\right)\right) \$
\end{aligned}
$$

where $A(I)(I:=1, \ldots$, DIMEVENV), $B(J)(J:=1, \ldots$, DIMODDV $)$ are elements of $C^{\infty}\left(\mathbb{R}^{m}\right) \otimes \Lambda(n)(2.1 .6)$ or

$$
\begin{aligned}
& \frac{\partial}{\partial r_{i}} \leftrightarrow D R \uparrow I \quad(i, I=1, \ldots, m), \\
& \frac{\partial}{\partial s_{j}} \leftrightarrow D S \uparrow J \quad(j, J=1, \ldots, n) .
\end{aligned}
$$

\section{EXAMPLE 2.2}

$$
\begin{aligned}
& v=f_{1} \frac{\partial}{\partial r_{1}}+\left(f_{2} s_{2}+f_{3} s_{1} s_{3}\right) \frac{\partial}{\partial s_{1}}+f_{4} \frac{\partial}{\partial s_{2}} \\
& V:=\mathrm{F}(1)^{*} \mathrm{DR}+\mathrm{F}(2)^{*} \mathrm{ALT}(2)^{*} \mathrm{DS}+\mathrm{F}(3)^{*} \mathrm{ALT}(1,3)^{*} \mathrm{DS}+\mathrm{F}(4)^{*} \mathrm{DS} * 2 \$
\end{aligned}
$$

where $\mathrm{F}(1), \ldots, F(4)$ are functions dependent of $\mathrm{R} 1, \ldots, R M$.

The choice for a bivariate polynomial was made to get a quick access to the components of the vector field by means of the procedure COEFF, and the sparse representation of polynomials. 
2.3. REPRESENTATION OF GRADED DIFFERENTIAL FORMS

A graded differential form $\beta \in \Omega\left(\mathbb{R}^{m}\right)$ has the representation

$$
\beta=\sum_{\mu, v} \mathrm{~d} r_{\mu} \mathrm{d} s^{v} f_{\mu, v},
$$

where

$$
\mu \in M_{n}, v=\left(v_{1}, \ldots, v_{n}\right) \in N^{n}, \quad f_{\mu, v} \in C^{\infty}\left(\mathbb{R}^{m}\right) \otimes \Lambda(n) .
$$

Note. In any case, the right module structure of $\Omega\left(\mathbb{R}^{m}\right)$ will be assumed and used.

A basic differential form $\mathrm{d} r_{\mu} \mathrm{d} s^{v}\left(\mu \in M_{n} ; v \in N^{n}\right)$ is represented as

$$
\mathrm{d} r_{\mu} \mathrm{d} s^{v} \leftrightarrow \operatorname{WEDGE}\left(\mu_{1}, \ldots, \mu_{k}, s_{1}, v_{1}, s_{2}, v_{2}, \ldots, s_{n}, v_{n}\right)
$$

under the following condition:

if $v_{i}=0$ then $s_{i}, v_{i}$ are omitted from the WEDGE representation;

if $v_{i}=1$ then $v_{i}$ is omitted.

\section{EXAMPLE 2.3}

$$
\mathrm{d} r_{1} \mathrm{~d} r_{3} \mathrm{~d} s_{1}^{2} \mathrm{~d} s_{3}^{3} \leftrightarrow \operatorname{WEDGE}\left(1,3, s_{1}, 2, s_{3}, 3\right) .
$$

We now start the description of the procedures.

\section{DER(FIELD,FUNCTION)}

The procedure constructs the action of a graded vector field (2.4) on a graded function (2.1.b), a derivation

\section{parameters:}

FIELD: the graded vector field FUNCTION: the graded function procedure calls:

OPCOEFF, MULFORM, B!@IP result:

The result is just the derivation of the function with respect to the field.

\section{EXAMPLE 2.4}

$$
\begin{aligned}
\mathrm{VEC}:= & \mathrm{DR}+\left(\mathrm{F}(3)^{*} \mathrm{ALT}(2)+\mathrm{F}(4)^{*} \mathrm{ALT}(1,3)\right)^{*} \mathrm{DS} \\
& +\mathrm{DS} \uparrow 2
\end{aligned}
$$

\section{EVENFP(FU)}

The procedure checks whether an element of $\Lambda(n)$ is even or odd. 
parameters:

FU: an element of $\Lambda(n)$; i.e. $s_{\mu}: \operatorname{ALT}\left(\mu_{1}, \ldots, \mu_{k}\right)$

result:

if $s_{\mu}$ even then 1 ( $k$ even)

else NIL.

\section{EVENODDPART(CO.ARCO)}

The procedure splits a graded function $\left(\epsilon C^{\infty}\left(\mathbb{R}^{m}\right) \otimes \Lambda(n)\right)$ in its even and odd part.

parameters:

$\mathrm{CO}:$ a graded function

ARCO: an array of size 2

procedure calls:

OPCOEFF, EVENFP

effect:

The even part of the graded function is stored as $\operatorname{ARCO}(0)$, while the odd part is stored as $\operatorname{ARCO}(1)$.

\section{$\operatorname{SUPCOM}(F, G)$}

The procedure constructs the graded Lie bracket (1.8) (or super commutator) of two graded vector fields $F$ and $G ; F, G \in \operatorname{Der}\left(C^{\infty}(U) \otimes \Lambda(n)\right)$

$[F, G]=F G-(-1)^{|F||G|} G F$

parameters:

$F$ : the first vector field

$G$ : the second vector field.

procedure calls:

DER, EVENODDPART

result:

The result is the graded Lie bracket of the vector fields $F$ and $G$.

\section{EVENWEDGEP(L)}

The procedure EVENWEDGEP calculates whether a basic differential form $\mathrm{d} r_{\mu} \mathrm{d} s^{v} \in \Omega\left(\mathbb{R}^{m}, C^{\infty}\left(\mathbb{R}^{m}\right) \otimes \Lambda(n)\right)$ is even or odd with respect to the first part of the bidegree $(b, j)=(l(\mu)+|v|,|v|)$.

parameters:

L: a basic differential form, WEDGE $\left(^{*}\right)$.

result:

The result of the procedure is

$\mathrm{T}$ : in case $l(\mu)+|v|$ is even $(0 \bmod 2)$

NIL: in case $l(\mu)+|y|$ is odd $(1 \bmod 2)$.

\section{CHANGESIGNP(I,L1,L2)}

The procedure CHANGESIGNP establishes a sign, which has to be taken care of in the construction of the multiplication in the bigraded algebra of differential forms $\Omega\left(\mathbb{R}^{m}\right)$. 
parameters:

I: any number (in application \pm 1 )

L1: $T$ or NIL

L2: T or NIL.

result:

\begin{tabular}{llll|rlll}
$i$ & L1 & L2 & result & $i$ & L1 & L2 & result \\
1 & T & NIL & NIL & -1 & T & NIL & T \\
1 & T & T & NIL & -1 & T & T & T \\
1 & NIL & NIL & T & -1 & NIL & NIL & NIL \\
1 & NIL & T & NIL & -1 & NIL & T & T
\end{tabular}

DERADALL(L1,L2)

The procedure DERADALL constructs the $s$-part of the product of two basic differential forms i.e.,

$\pm s^{v_{1}+v_{2}}=s^{v_{1}} \cdot s^{v_{2}}\left(v_{1}, v_{2} \in N^{n}\right)$.

parameters:

L1: the first list, associated to $s^{v_{1}}$

L2: the second list, associated to $s^{v_{2}}$.

result:

the list associated to $s^{v_{1}+v_{2}}$.

Note: the procedure DERADALL is an extension of the procedure DERAD, available in REDUCE, and used in the representation of higher-order derivatives of functions.

\section{EXAMPLE 2.5}

$$
\begin{array}{r}
\text { LISP } ; L 1:='\left(\begin{array}{llll}
s 1 & 2 & s & s 4
\end{array}\right) \$ \\
\text { L1 }:='\left(\begin{array}{llll}
s 2 & s & s & 2
\end{array}\right) \$
\end{array}
$$

DERADALL(L1, L2)

(S1 2 S2 2 S3 S4 3).

\section{SPLITWEDGE(A)}

The procedure SPLITWEDGE splits a basic differential form $\mathrm{d} r_{\mu} \mathrm{d} s^{\nu}\left(\mu \in M_{n}, v \in N^{n}\right)$ represented as WEDGE $\left({ }^{*}\right)$ in the ' $\mathrm{d} r_{\mu}{ }^{\prime}$ ' (even) and ' $\mathrm{d} s^{v}{ }$ ' (odd) parts.

parameters:

A: the basic differential form: WEDGE $\left({ }^{*}\right)$.

result:

the result of the procedure SPLITWEDGE is a list, which CAR is the list associated to $\mathrm{d} r_{\mu}$, the CDR being the list associated to $\mathrm{d} s^{v}$.

EXAMPLE 2.6

$$
\begin{gathered}
\text { lisp; } 11:=\text { ' (wedge } 135 \mathrm{~s} 1 \mathrm{~s} 23 \mathrm{~s} 3) \$ \\
11 \mathrm{a}:=\operatorname{splitwedge}(11) ;
\end{gathered}
$$


((1 3 5) S1 S2 3 S3)

car 11a;

$\left(\begin{array}{lll}1 & 3 & 5\end{array}\right)$

cdr 11a;

(S1 S2 3 S3).

\section{BASICSUPMUL(W1, W2, FAKW1, FAKW2)}

The procedure BASICSUPMUL constructs the multiplication of two graded differential forms W1*FAKW1 and W2*FAKW2 in the bigraded algebra of differential forms $\Omega\left(\mathbb{R}^{m}, C^{\infty}\left(\mathbb{R}^{m}\right) \otimes \Lambda(n)\right)$, whereas each differential form consists of only one term.

\section{parameters:}

W1: the basic differential form associated to W1*FAKW1.

W2: the basic differential form associated to W2*FAKW2.

FAKW1: the coefficient of W1*FAKW1 $\left(\right.$ FAKW1 $\left.\in C^{\infty}\left(\mathbb{R}^{m}\right) \otimes \Lambda(n)\right)$.

FAKW2: the coefficient of W2*FAKW2 (FAKW $\left.2 \in C^{\infty}\left(\mathbb{R}^{m}\right) \otimes \Lambda(n)\right)$.

procedure calls:

SPLITWEDGE, MERGESTRICT [1], EVENWEDGEP, MULFORM [1] EVENODDPART, CHANGESIGNP, DERADALL

result:

the result is the graded (super) multiplication of two oneterm differential forms in the right $C^{\infty}\left(\mathbb{R}^{m}\right) \otimes \Lambda(n)$-module representation of $\Omega\left(\mathbb{R}^{m}, C^{\infty}\left(\mathbb{R}^{m}\right) \otimes \Lambda(n)\right)$.

\section{EXAMPLE 2.7}

$$
\begin{aligned}
& 11:=\text { wedge }(1,2, \text { s1, 2)\$ } \\
& 111:=\operatorname{alt}(1) \$ \\
& 12:=\text { wedge(s2)\$ } \\
& 112:=3^{*} \mathrm{r}_{1}{ }^{* *} 2(\operatorname{alt}(1)+\operatorname{alt}(2)) \$ \\
& \text { basicsupmul( } 11,12,111,112) \\
& \text { WEDGE }(1,2, \mathrm{~S} 1,2, \mathrm{~S} 2)^{*}\left(-3^{*} \mathrm{R} 11^{*} 2^{*} \mathrm{ALT}(1,2)\right)
\end{aligned}
$$

\section{SUPMUL(A,B)}

The procedure SUPMUL constructs the graded multiplication of two general differential forms, and is actually a repeated application of the procedure BASICSUPMUL.

parameters:

A: the first differential form.

B: the second differential form.

procedure call:

OPCOEFF, BASICSUPMUL.

result:

the graded multiplication of two graded differential forms.

\section{SUPDF(W)}

In general, a graded differential form is a sum of terms, where each of them is a 
product of a basic graded form with a graded function. Therefore, the procedure SUPDF splits a general form into its constituent parts and constructs the result by summing up the various graded exterior derivatives of all terms.

This procedure returns the graded exterior derivative of $\mathrm{W}$.

parameter:

W: a graded differential form.

procedure calls:

BASICSUPDF, EMPTYWEDGE, OPCOEFF

result:

The graded exterior derivative of $\mathrm{W}$ is returned as the result.

\section{BASICSUPDF(W,F)}

This procedure computes the exterior derivative of one term, which has already been split into a form and function factor.

parameter:

W: a basic graded differential form

F: a graded function.

procedure calls:

EVENWEDGEP, GETFIRSTOP, SPLITWEDGE

result:

The graded exterior derivative of $W * F$.

Note: The order of the parameters $\mathrm{W}$ and $\mathrm{F}$ are according to the convention, that we consider the forms as a right module.

\section{EMPTYWEDGE( )}

This procedure returns the identity as a graded form, depending on the value of SUPMULNAME.

result:

One as a zeroform. For example WEDGE( ), assuming that SUPMULNAME holds the value 'WEDGE'.

EXAMPLE 2.8

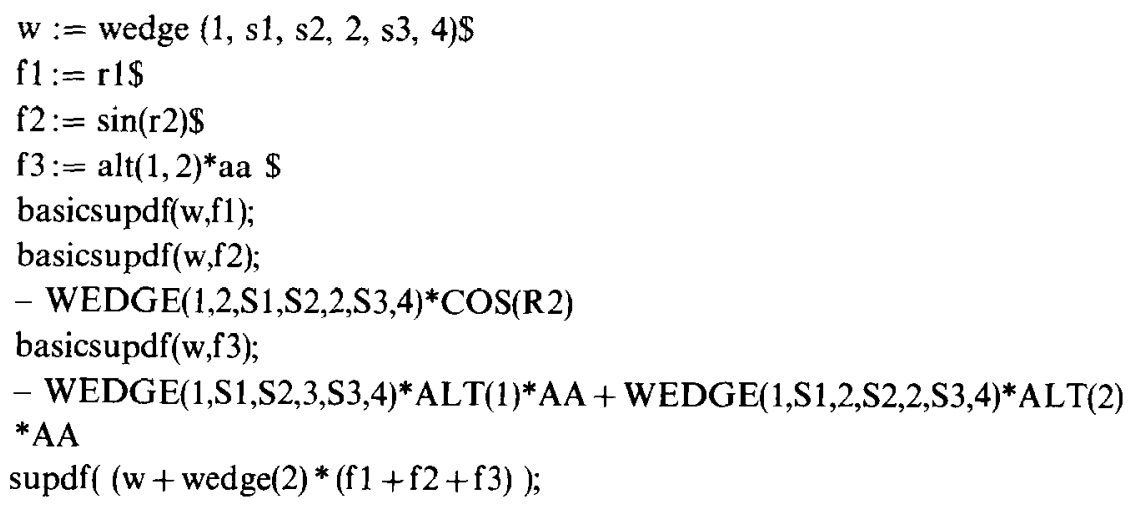


- WEDGE(2,S1)*ALT(2)*AA + WEDGE(2,S2)*ALT(1)*AA - WEDGE(1,S1, S2,3,S3,4)*ALT(1)*AA + WEDGE(1,S1,2,S2,2,S3,4)*ALT(2)*AA - WEDGE(1, $2, \mathrm{~S} 1, \mathrm{~S} 2,2, \mathrm{~S} 3,4)^{*} \mathrm{COS}(\mathrm{RS})+\operatorname{WEDGE}(1,2)$

\section{SUPIP(F,SW)}

To implement the graded contraction, the 'even and odd parts' are done separately by two procedures. Furthermore, some help functions are introduced. This is the general procedure for computing the inner product of graded field with a graded differential form.

parameters:

$F:$ the vector field

SW: the differential form

procedure calls:

BASICSUPIPEVEN, BASICSUPIPODD, OPCOEFF

result:

The inner product of the vector field and the differential form.

\section{BSUPIP(I,L)}

This is a function, which is used in computing the contribution of even basic vector fields.

\section{parameters:}

I: an integer $(1 \leqslant \mathrm{I} \leqslant$ DIMEVEN).

$\mathrm{L}$ : a list of integers (representing the even part of a differential form). result:

Either NIL is returned, if I does not occur in L or a list of integers. The first element is either +1 or -1 , followed by the elements of $\mathrm{L}$, where $\mathbf{I}$ is deleted. The sign of the first element depends on the location of $\mathrm{I}$ in $\mathrm{L}$, an odd place gives a +1 , an even place gives a -1 .

\section{CHANGEFORSUPIP(NAME)}

This is a function to get a better performance of SUPIP. The argument of a basic form consists, in general, of an even part, a list of positive integers, and an odd part, a list of odd variable - names possibly followed immediately by a positive integer, combined into one list. This list has to be split into its odd and even part for each term of an inner product. These lists are, in fact, stored under the PROPERTY!@OPELEMENT of the parameter NAME, where NAME has been a third parameter of OPCOEFF, namely to decompose the differential form of an inner product operation.

If this splitting is done once and for all, the procedure will have a better performance.

\section{parameter:}

NAME: an identifier, which should have been used at least once by OPCOEFF as the third parameter.

result: 
This function is called for its side effect, each element of the first element of the list stored under the PROPERTY!@OPELEMENT of NAME will be replaced by the result of the action of the function SPLITWEDGE.

EXAMPLE 2.9

opcoeff(wedge(1) + wedge(s2) + wedge( $1,2, \mathrm{~s} 1, \mathrm{~s} 2,4)$, wedge,cw)\$

lisp mapcar(car get('cw,!! opelement),'print)\$

(WEDGE 1)

(WEDGE 12 S1 2 S2 4)

(WEDGE S2)

changeforsupip(cw)\$

input 3;

((1 2) S1 2 S2 4)

(NIL S2)

\section{BASICSUPIPEVEN(I,F1,J,F2)}

This function computes the contribution of even basic vector fields from an inner product, it works 'only' in conjunction with SUPIP.

parameters:

I : an integer, the I-th even basic vector field.

F1: the coefficient of the I-th even basic vector field.

$\mathrm{J}$ : an integer, which points to J-th basic differential form from the input of SUPIP.

F2: the coefficient of the J-th basic differential form from the input of SUPIP. result:

The inner product of one fieldterm with one formterm.

procedure calls:

BSUPIP, EVENWEDGEP, EVENODDPART, MULFORM

\section{BASICSUPIPODD(I,F1,J,F2)}

The description is analogous to BASICSUPIPEVEN, the only difference is that odd basic vector fields are used, coming from the input of SUPIP.

\section{INVDERADD(VAR,L)}

This function is used to compute the shortened list of a basic differential form, if a partial contribution unequal to $\varnothing$ has to be computed, reflecting the fact that the order of the differential form after an inner product is reduced by one.

parameters:

VAR: an identifier, the name of an odd variable.

L : a list, where VAR must be present (precisely) once.

result:

A 'shortened' list L. 
EXAMPLE 2.10

invderad('s1,'(1 2

(1 $\left.\begin{array}{lll}1 & 3\end{array}\right)$

invderad('s1,'(1 s1 4));

(1 S1 3).

\section{Nonlocal Symmetries and Hierarchies of (Super)Symmetries of the Supersymmetric mKdV Equation}

As an application of the developed software described in the preceding sections, we discuss the Lie algebraic structure of the supersymmetric modified $\mathrm{KdV}$ equation

$$
v_{t}=-v_{3}+6 v^{2} v_{1}-3 \varphi\left(v \varphi_{1}\right)_{1}, \quad \varphi_{t}=-\varphi_{3}+3 v(v \varphi)_{1},
$$

whereas in (3.1) the integer indices refer to differentiation with respect to $x$, i.e. $v_{1}=v_{x}$, $v_{3}=v_{x x x} ; x, t, v$ are even, while $\varphi$ is an odd variable [11].

Putting $\varphi=0$ in (3.1), we return to the classical case. Classical higher-order symmetries are vector fields defined on the infinite jet bundle $J^{\infty}(x, t ; v, \varphi)$ and which satisfy the symmetry condition

$$
\mathscr{L}_{V}\left(D^{\infty} I\right) \subset D^{\infty} I,
$$

where $D^{\infty} I$ denotes the infinite prolongation of the exterior differential system $I$ describing the partial differential equation at hand by means of the action of the total partial derivative vector field $D_{x}, D_{t}$, defined by

$$
D_{x}=\partial_{x}+u_{x} \partial_{u}+\varphi_{x} \partial_{\varphi}+\cdots, \quad D_{t}=\partial_{t}+u_{t} \partial_{u}+\varphi_{t} \partial_{\varphi}+\cdots
$$

In (3.2), $\mathscr{L}_{V}$ denotes the Lie derivative by the vector field $V$. Due to the fact that (3.3) satisfies (3.2) in an obvious way, we restrict our search for symmetries to vertical vector fields, i.e. the components $\partial_{x}, \partial_{t}$ are taken to be zero.

Vertical vector fields have been proved to have the following representation

$$
V=f \partial_{v}+g \partial_{\varphi}+\left(D_{x} f\right) \partial_{v_{1}}+\left(D_{x} g\right) \partial_{\varphi_{1}}+\cdots,
$$

so we are only interested in the generating functions [15], $f, g$ of the vector field. The functions $f, g$ are assumed to depend on a finite number of variables of $J^{\infty}(x, t ; v, \varphi)$.

In the graded case at hand, we proceed in a similar way, keeping in mind the left module structure of vector fields.

We restrict our search for higher-order symmetries at this moment to even vector fields.

Equation (3.1) is graded in the classical sense, i.e.

$$
\begin{array}{lll}
\operatorname{deg}(x)=-1, & \operatorname{deg}(v)=1, & \operatorname{deg}\left(v_{1}\right)=2, \\
\operatorname{deg}(t)=-3, & \operatorname{deg}(\varphi)=\frac{1}{2}, & \operatorname{deg}\left(\varphi_{1}\right)=1 \frac{1}{2},
\end{array}
$$

so each term in (3.1) is of degree 4 and $3 \frac{1}{2}$, respectively. Our search is for even vector 
fields whose defining functions $f, g$ depend on

$$
\varphi, v, \varphi_{1}, v_{1}, \ldots, \varphi_{5}, v_{5},
$$

more specifically,

$$
\begin{aligned}
& f=f_{1}+f_{2} \varphi \varphi_{5}+f_{3} \varphi \varphi_{4}+f_{4} \varphi_{2} \varphi_{3}+f_{5} \varphi_{1} \varphi_{3}+f_{6} \varphi \varphi_{3}+f_{7} \varphi_{1} \varphi_{2}+f_{8} \varphi \varphi_{2} \\
& g=g_{1} \varphi_{5}+g_{2} \varphi_{4}+g_{3} \varphi_{3}+g_{4} \varphi \varphi_{1} \varphi_{3}+g_{5} \varphi_{2}+g_{6} \varphi \varphi_{1} \varphi_{2}+g_{7} \varphi_{1}+g_{8} \varphi
\end{aligned}
$$

whereas in (2.6) $f_{1}, \ldots, f_{8}, g_{1}, \ldots, g_{8}$ are dependent on the even variables $v, \ldots, v_{5}$.

The vector field $V(3.4),(3.6)$ has to satisfy the symmetry condition (3.2) which is equivalent to

$$
\mathscr{L}_{V}\left(v_{t}+v_{2}-6 v^{2} v_{1}+3 \varphi(v \varphi)_{1}\right) \doteq 0, \quad \mathscr{L}_{V}\left(\varphi_{t}+\varphi_{2}-3 v(v \varphi)_{1}\right) \doteq 0,
$$

where ' $\doteq 0$ ' is to be understood as equal to zero on the submanifold of the infinite jet bundle $J^{\infty}(x, t ; v, \varphi)$ defined by (3.1) and its differential consequences.

Conditions (3.7) lead to an overdetermined system of partial differential equations for the functions $f_{1}, \ldots, f_{8}, g_{1}, \ldots, g_{8}$, using the described software for graded differential geometry.

Solving the resulting system of equations [2], we arrive at the following result.

THEOREM 1. The supersymmetric modified KdV equation (3.1) admits the following even symmetries arising from (3.6)

$$
\begin{aligned}
X_{1}= & v_{1} \partial_{v}+\varphi_{1} \partial_{\varphi}, \\
X_{3}= & \left(-v_{3}+6 v^{2} v_{1}-3 v \varphi \varphi_{2}-3 v_{1} \varphi \varphi_{1}\right) \partial_{v}+\left(-\varphi_{3}+3 v v_{1} \varphi+3 v^{2} \varphi_{1}\right) \partial_{\varphi}, \\
X_{5}= & \left(v_{5}-10 v_{3} v^{2}-40 v_{2} v_{1} v-10 v_{1}^{3}+30 v_{1} v^{4}+\right. \\
& +5 v \varphi \varphi_{4}+10 v_{1} \varphi \varphi_{3}+5 v \varphi_{1} \varphi_{3}+5 v_{1} \varphi_{1} \varphi_{2}-20 v^{3} \varphi \varphi_{2}+ \\
& \left.+10 v_{2} \varphi \varphi_{2}+5 v_{3} \varphi \varphi_{1}-60 v_{1} v^{2} \varphi \varphi_{1}\right) \partial_{v}+ \\
& +\left(\varphi_{5}-5 v^{2} \varphi_{3}-15 v_{1} v \varphi_{2}-15 v_{2} v \varphi_{1}-10 v_{1}^{2} \varphi_{1}+10 v^{4} \varphi_{1}-\right. \\
& \left.-5 v_{3} v \varphi-10 v_{2} v_{1} \varphi+20 v_{1} v^{3} \varphi\right) \partial_{\varphi} .
\end{aligned}
$$

Note. Of course, (3.1) admits the scaling symmetry which is equivalent to

$$
-x \partial_{x}-3 t \partial_{t}+v \partial_{v}+\frac{1}{2} \varphi \partial_{\varphi}
$$

due to (3.5).

In a similar way as in the even case, we searched for odd symmetries and arrived at the following odd or supersymmetry

$$
Y_{1 / 2}=\varphi_{1} \partial_{v}+v \hat{\partial}_{\varphi}
$$

and there exist no other (local) higher-order odd symmetries. 
Motivated by results obtained for the super $\mathrm{KdV}$ equation [12] we introduce nonlocal variables in order to construct a nonlocal symmetry of (3.1).

First note that

$$
q_{1 / 2}=\int_{-\infty}^{x} v \varphi \mathrm{d} x
$$

is a potential of $(3.1)$, i.e.

$$
\begin{aligned}
\left(q_{1 / 2}\right)_{x} & =v \varphi \\
\left(q_{1 / 2}\right)_{t} & =\int_{-\infty}^{x} v_{t} \varphi+v \varphi_{t} \mathrm{~d} x \\
& =\int_{-\infty}^{x}\left(-v_{3}+6 v^{2} v_{1}-3 v \varphi \varphi_{2}-3 v_{1} \varphi \varphi_{1}\right) \varphi+v\left(-\varphi_{3}+3 v(v \varphi)_{1}\right) \\
& =-v_{2} \varphi+v_{1} \varphi_{1}-v \varphi_{2}+3 v^{3} \varphi
\end{aligned}
$$

and

$$
Q_{1 / 2}=\int_{-\infty}^{\infty} v \varphi \mathrm{d} x
$$

is a conserved quantity of (3.1).

We make the following observation.

THEOREM 2. The vector field $Z_{1}$, defined by

$$
q_{1 / 2} \varphi_{1} \partial_{v}+\left(q_{1 / 2} v-\varphi_{1}\right) \partial_{\varphi}
$$

is a nonlocal symmetry of (3.1).

Proof. In order to prove that a vector field $V$

$$
V=V_{v} \partial_{v}+V_{\varphi} \partial_{\varphi}
$$

is a nonlocal symmetry of (3.1), we have to take the prolongation of the total partial derivative vector fields $D_{x}, D_{t}$ towards to nonlocal variables, i.e.

$$
\tilde{D}_{x}=D_{x}+(v \varphi) \partial_{q_{1 / 2}}, \quad \tilde{D}_{t}=\left(-v_{2} \varphi+v_{1} \varphi_{1}-v \varphi_{2}+3 v^{3} \varphi\right) \partial_{q_{1 / 2}}
$$

then the symmetry condition

$$
\begin{aligned}
& \mathscr{L}_{V}\left(-v_{t}-v_{3}+6 v^{2} v_{1}-3 v \varphi \varphi_{2}-3 v_{1} \varphi \varphi_{1}\right)=0 \\
& \mathscr{L}_{V}\left(-\varphi_{t}-\varphi_{3}+3 v^{2} \varphi_{1}+3 v v_{1} \varphi\right)=0
\end{aligned}
$$

can be written as [15]

$$
\begin{aligned}
& -\tilde{D}_{t}\left(V_{v}\right)-\left(\tilde{D}_{x}\right)^{3}\left(V_{v}\right)+12 v\left(V_{v}\right) v_{1}+6 v^{2} \tilde{D}_{x}\left(V_{v}\right)-3 V_{v} \varphi \varphi_{2}- \\
& -3 v V_{\varphi} \varphi_{2}-3 v \varphi\left(\tilde{D}_{x}\right)^{2}\left(V_{\varphi}\right)-3 \tilde{D}_{x}\left(V_{v}\right) \varphi \varphi_{1}-3 v_{1} V_{\varphi} \varphi_{1}-3 v_{1} \varphi \tilde{D}_{x}\left(V_{\varphi}\right)=0
\end{aligned}
$$


and

$$
\begin{aligned}
& -\tilde{D}_{t}\left(V_{\varphi}\right)-\left(\tilde{D}_{x}\right)^{3}\left(V_{\varphi}\right)+6 v V_{v} \varphi_{1}+3 v^{2} \tilde{D}_{x}\left(V_{\varphi}\right)+3 V_{v} \varphi+ \\
& \quad+3 v \tilde{D}_{x}\left(V_{v}\right) \varphi+3 v v_{1} \tilde{D}_{x}\left(V_{\varphi}\right)=0 .
\end{aligned}
$$

Substitution of $V_{v}=q_{1 / 2} \varphi_{1}$ and $V_{\varphi}=q_{1 / 2} v-\varphi_{1}$ into (3.17a) and (3.17b) yields, after a tedious calculation, that (3.16) is satisfied by (3.13).

In a way similar to the super $\mathrm{KdV}$ equation [13], we construct a hierarchy of nonlocal odd symmetries $\left(Y_{n+(1 / 2)}\right)_{n \in \mathbb{N}}$ by defining

$$
Y_{n+(3 / 2)}=\left[Z_{1}, Y_{n+(1 / 2)}\right] \quad(n \in \mathbb{N}),
$$

whereas the even hierarchy $X_{1}, X_{3}, X_{5}, \ldots,\left(X_{n+1}\right)$ results from

$$
X_{2 n+1}=2\left[Y_{n+(1 / 2)}, Y_{n+(1 / 2)}\right] \quad(n \in \mathbb{N}) .
$$

Moreover, the even and odd potentials $p_{1}, p_{3}, \ldots, q_{1 / 2}, q_{3 / 2}, q_{5 / 2}, \ldots$ leading to even and odd conserved quantities, result from the construction of the graded Lie algebra (3.18), (3.19) by prolongation of the vector fields towards nonlocal variables.

We now present the even potentials $p_{1}, p_{3}$ and the nonlocal superpotentials $q_{1 / 2}$, $q_{3 / 2}, q_{5 / 2}$, the quantities being $P_{1}, P_{3}, Q_{1 / 2}, Q_{3 / 2}, Q_{5 / 2}$.

$$
\begin{aligned}
& p_{1}=\int_{-\infty}^{x}\left(v^{2}-\varphi \varphi_{1}\right) \mathrm{d} x, \quad p_{3}=\int_{-\infty}^{x}\left(-v^{4}-v_{1}^{2}+3 \varphi \varphi_{1} v^{2}+\varphi_{1} \varphi_{2}\right) \mathrm{d} x, \\
& q_{1 / 2}=\int_{-\infty}^{x}(v \varphi) \mathrm{d} x, \quad q_{3 / 2}=\int_{-\infty}^{x}\left(p_{1} v \varphi+v \varphi_{1}\right) \mathrm{d} x, \\
& q_{5 / 2}=\int_{-\infty}^{x}\left(-\left(p_{1}\right) v^{2} \varphi-2 p_{1} v \varphi_{1}+v^{3} \varphi-2 v \varphi_{2}\right) \mathrm{d} x .
\end{aligned}
$$

The $x$-derivatives are just the integrands in (3.20), (3.21) while the $t$-derivatives are given by

$$
\begin{aligned}
\left(p_{1}\right)_{t}= & 3 v^{4}+v_{1}^{2}-2 v v_{2}-9 v^{2} \varphi \varphi_{1}+\varphi \varphi_{3}-2 \varphi_{1} \varphi_{2}, \\
\left(p_{3}\right)_{t}= & -4 v^{6}+4 v_{2} v^{3}-v_{2}^{2}+2 v_{1} v_{3}-12 v^{2} v_{1}^{2}+21 v^{4} \varphi \varphi_{1}- \\
& -9 v v_{2} \varphi \varphi_{1}+3 v_{1}^{2} \varphi \varphi_{1}+12 v v_{1} \varphi \varphi_{2}-3 v^{2} \varphi \varphi_{3}+ \\
& +9 v^{2} \varphi_{1} \varphi_{2}-\varphi_{1} \varphi_{4}+2 \varphi_{2} \varphi_{3}, \\
\left(q_{3 / 2}\right)_{t}= & p_{1}\left(-v_{2} \varphi+v_{1} \varphi_{1}-v \varphi_{2}+3 v^{3} \varphi\right)+2 v^{2} v_{1} \varphi- \\
& -v_{2} \varphi_{1}+4 v^{3} \varphi_{1}+v_{1} \varphi_{2}-v \varphi_{3}, \\
\left(q_{5 / 2}\right)_{t}= & -p_{1}^{2}\left(-v_{2} \varphi+v_{1} \varphi_{1}-v \varphi_{2}+3 v^{3} \varphi\right)+ \\
& +p_{1}\left(2 v \varphi_{3}-2 v_{1} \varphi_{2}-8 v^{3} \varphi_{1}+2 v_{2} \varphi_{1}-4 v^{2} v_{1} \varphi\right)+ \\
& +2 v \varphi_{4}-2 v_{1} \varphi_{3}-9 v^{3} \varphi_{2}+2 v_{2} \varphi_{2}-13 v^{2} v_{1} \varphi_{1}+ \\
& +4 v \varphi \varphi_{1} \varphi_{2}+5 v^{5} \varphi-9 v^{2} v_{2} \varphi .
\end{aligned}
$$


The symmetries obtained are given by

$$
\begin{aligned}
Z_{1}= & \left(q_{1 / 2} \varphi_{1}\right) \partial_{v}+\left(q_{1 / 2} v-\varphi_{1}\right) \partial_{\varphi}+\left(q_{1 / 2} v \varphi+\varphi \varphi_{1}\right) \partial_{p_{1}}+ \\
& +\left(q_{1 / 2} p_{1}-q_{3 / 2}\right) \partial_{q_{1 / 2}}, \\
Y_{1 / 2}= & \varphi_{1} \partial_{v}+v \partial_{\varphi}+v \varphi \partial_{p_{1}}+p_{1} \partial_{q_{1 / 2}}, \\
Y_{3 / 2}= & \left(2 q_{1 / 2} v_{1}-p_{1} \varphi_{1}+v^{2} \varphi-\varphi_{2}\right) \partial_{v}+\left(2 q_{1 / 2} \varphi_{1}-p_{1} v+v_{1}\right) \partial_{\varphi}+ \\
& +\left(2 q_{1 / 2}\left(v^{2}-\varphi \varphi_{1}\right)-p_{1} v \varphi-2 v \varphi_{1}+v_{1} \varphi\right) \partial_{p_{1}}+ \\
& +\left(2 q_{1 / 2} v \varphi-\frac{1}{2}\left(p_{1}\right)^{2}+\frac{1}{2} v^{2}+\varphi \varphi_{1}\right) \partial_{q_{1 / 2}}, \\
Y_{5 / 2}= & \left\{\frac{1}{2}\left(p_{1}\right)^{2} \varphi_{1}+p_{1}\left(\varphi_{2}-v^{2} \varphi\right)-2 q_{3 / 2} v_{1}+\varphi_{3}-\frac{5}{2} v^{2} \varphi_{1}-3 v v_{1} \varphi\right\} \partial_{v}+ \\
& +\left\{\frac{1}{2}\left(p_{1}\right)^{2} v+p_{1}\left(-v_{1}\right)-2 q_{3 / 2} \varphi_{1}+v_{2}-\frac{3}{2} v^{3}+2 v \varphi \varphi_{1}\right\} \partial_{\varphi}+ \\
& +\left\{\frac{1}{2}\left(p_{1}\right) v^{2} \varphi+p_{1}\left(2 v \varphi_{1}-v_{1} \varphi\right)-2 q_{3 / 2}\left(v^{2}-\varphi \varphi_{1}\right)+2 v \varphi_{2}-2 v_{1} \varphi_{1}+\right. \\
& \left.+v_{2} \varphi-\frac{7}{2} v^{3} \varphi\right\} \partial_{p_{1}}+ \\
& +\left\{\frac{1}{8}\left(p_{1}\right)^{4}-\frac{1}{4}\left(p_{1}\right)^{2}\left(+v^{2}+4 \varphi \varphi_{1}\right)-2 p_{1} q_{3 / 2} v \varphi-p_{1} \varphi \varphi_{2}-\right. \\
& \left.-2 q_{3 / 2} v \varphi_{1}-\varphi_{1} \varphi_{2}+v^{2} \varphi \varphi_{1}-\frac{11}{8} v^{4}+v v_{2}-\frac{1}{2} v_{1}^{2}\right\} \partial_{q_{3 / 2}}, \\
X_{1}= & v_{1} \partial_{v}+\varphi_{1} \partial_{\varphi}, \\
X_{3}= & \left(-v_{3}+6 v^{2} v_{1}-3 v \varphi \varphi_{2}-3 v_{1} \varphi \varphi_{1}\right) \partial_{v}+ \\
& +\left(-\varphi_{3}+3 v^{2} \varphi_{1}+3 v v_{1} \varphi\right) \partial_{\varphi}, \\
X_{5}= & \left(v_{5}-10 v_{3} v^{2}-40 v_{2} v_{1} v-10 v_{1}^{3}+30 v_{1} v^{4}+\right. \\
& +5 v \varphi \varphi_{4}+10 v_{1} \varphi \varphi_{3}+5 v \varphi_{1} \varphi_{3}+5 v_{1} \varphi_{1} \varphi_{2}-20 v^{3} \varphi \varphi_{2}+ \\
& \left.+10 v_{2} \varphi \varphi_{2}+5 v_{3} \varphi \varphi_{1}-60 v_{1} v^{2} \varphi \varphi_{1}\right) \partial_{v}+ \\
+ & \left(\varphi_{5}-5 v^{2} \varphi_{3}-15 v_{1} v \varphi_{2}-15 v_{2} v \varphi_{1}-10 v_{1}^{2} \varphi_{1}+10 v^{4} \varphi_{1}-\right. \\
& \left.-5 v_{3} v \varphi-10 v_{2} v_{1} \varphi+20 v_{1} v^{3} \varphi\right) \partial_{\varphi} .
\end{aligned}
$$

The graded Lie algebra structure is similar to the structure obtained for the $\mathrm{KdV}$ equation [13].

\section{Conclusion}

We discussed the construction of a graded differential geometry package which has already turned out to be very useful in the study of supersymmetric equations in mathematical physics. As an application, we studied here symmetry structures of the Manin-Radul supersymmetric extension of the modified $\mathrm{KdV}$ equation.

\section{References}

1. Gragert, P. K. H.: Symbolic computations in prolongation theory, PhD thesis, University of Twente (1981). 
2. Kersten, P. H. M.: Infinitesimal symmetries: A computational approach, CWI tract 17, Center for Mathematical and Computer Science, Amsterdam (1987);

Kersten, P. H. M.: Software to compute infinite symmetries of exterior differential systems, with applications, Acta Appl. Math. 16 (1989), 207.

3. Schwarz, F.: Symmetries of differential equations: from Sophus Lie to compute algebra, SIAM Rev. 30 (1988), 450-481.

4. Hearn, A. C.: REDUCE User's Manual (Version 3.0), The Rand Corporation, Santa Monica (1983).

5. Reid, G. J.: Finding symmetries of differential equations without integrating determining equations, submitted to European J. Appl. Math. (1990).

6. Champagne, B., Hereman, W., and Winternitz, P.: The computer calculations of Lie point symmetries of large systems of differential equations, submitted to Comput. Phys. Comm. (1990).

7. Wess, J. and Zumino, B.: Supergauge transformation in four dimensions, Nuclear Phys. B70 (1974), 3950 ;

Wess, J. and Zumino, B.: A Lagrangian model invariant under supergauge transformations, Phys. Lett. 49B (1974), 52-54.

8. Kostant, B.: Graded manifolds, graded Lie theory and prequantization, in Differential Geometrical Methods in Mathematical Physics (Bonn 1975), Lecture Notes in Mathematics 570, Springer, New York (1977), pp. 177-306.

9. Gragert, P. K. H. and Kersten, P. H. M.: Graded differential geometry in REDUCE 3, Memorandum 680, Department of Applied Mathematics, University of Twente, Enschede, The Netherlands (1988).

10. Kersten, P. H. M. and Gragert, P. K. H.: Symmetries for super KdV-equation, Memorandum 681, Department of Applied Mathematics, University of Twente, Enschede, The Netherlands (1988);

Kersten, P. H. M. and Gragert, P. K. H.: J. Phys. A 21 (1988), L579-584.

11. Mathieu, P.: Supersymmetric extension of the Korteweg-de Vries equation, J. Math. Phys. 23 (1988), 2499-2506.

12. Manin, Yu. I. and Radul, A. O.: A supersymmetric extension of the Kadomtsev-Petviashvi hierarchy, Comm. Math. Phys. 98 (1985), 65.

13. Kersten, P. H. M.: Higher order sypersymmetries and fermionic conservation laws of the supersymmetric extension of the KdV equation, Phys. Lett. 134A (1988), 25-30.

14. Roelofs, G. H. M. and Hijligenberg, H. van de: Prolongation structures for supersymmetric equations, J. Phys. A 23 (1990).

15. Roelofs, G. H. M. and Kersten, P. H. M.: Supersymmetric extensions of the nonlinear Schrödinger equation: symmetries and coverings, Department of Applied Mathematics, University of Twente, Enschede, The Netherlands (1990).

16. Krasilshchik, I. S., Lychagin, V. V. and Vinogradov, A. M.: Geometry of Jetspaces and Nonlinear Partial Differential Equations, Adv. Stud. Contemp. Math. Vol. 1, Gordon \& Breach, New York (1986). 